\title{
Pescadores Artesanales y Desarrollo Turístico del Borde Costero de Atacama, Chile ${ }^{1}$
}

\author{
Local Fishermen and Tourism Development \\ in Atacama's Coastal Area, Chile
}

\section{Enzo Acuña $a^{2}$ \\ Gabriel Canihuante \\ Sergio Zúñiga ${ }^{4}$}

\begin{abstract}
RESUMEN: Los pescadores artesanales de la Región de Atacama, en el centronorte de Chile, ven en el turismo una posibilidad de desarrollo complementario a sus principales formas de vida: la captura y venta de productos del mar. A partir de un estudio solicitado por las autoridades de la región (Sistema Desarrollo del Borde Costero, Pesca Artesanal y Turismo) se realizó un diagnóstico de la situación del turismo en el $\mathrm{BCA}$, desde la óptica de los pescadores, y se elaboró una serie de propuestas posibles de llevar a cabo por este sector. En este documento se presenta un resumen de la parte correspondiente al turismo en dicho estudio, realizado por encargo del Gobierno Regional de Atacama.
\end{abstract}

PALABRAS-CLAVE: Sistema Borde Costero; pesca artesanal; Región de Atacama, Chile; propuestas de intervención; desarrollo turístico.

ABSTRACT: The local fishermen from the Atacama Region, located in northern Chile, understand that tourism is a great possibility for them to

1. Versión parcial del Estudio Sistema Desarrollo del Borde Costero (Pesca Artesanal y Turismo), financiado por el Servicio de Cooperación Técnica (SERCOTEC) Región de Atacama. 2002-2003.

2. Médico veterinario. Magister en Ciencias y académico de la Universidad Católica del Norte, sede Coquimbo. Contacto: eacuna@ucn.cl.

3. Periodista. Magister en Ciencias Geográficas y académico de la Universidad del Mar, sede La Serena. Contacto: gcanihuante@udelmar.cl.

4. Ingeniero comercial. Magister en Economía y académico de la Universidad Católica del Norte, sede Coquimbo. Contacto: szuniga@ucn.cl. 
develop new economic activities different from fishing. Local people proposed different and possible new business activities in the area, where different tourist variables were evaluated. The proposal is based on a study carried out by authorities from the Atacama Region about the development of the coast of the area, local fishing and tourism altogether. This document is a summary of the study above.

KEYWORDS: coastal system; local fishing; Atacama Region, Chile; tourism related proposals; tourist development.

\section{Presentación}

En septiembre de 2002 un equipo interdisciplinario de profesionales de la Universidad Católica del Norte - UCN sede Coquimbo, se adjudicó un proyecto de estudio en una licitación convocada por el estatal Servicio de Cooperación Técnica-SERCOTEC de la Región de Atacama, para la "Elaboración del Diagnóstico, Línea Base, Identificación de Oportunidades y Plan de Intervención (2003-2005) - Sistema Desarrollo del Borde Costero (Pesca Artesanal y Turismo)", el que fue concluido a mediados de 2003.

El objetivo fundamental de este estudio era presentar al Gobierno regional, vía SERCOTEC, una propuesta de Plan de Intervención con inversiones en proyectos que la población de los pescadores artesanales pudieran llevar a cabo, mediante sus propias organizaciones, de modo que mejoren su calidad de vida, generando nuevas alternativas productivas, y aumentando la eficiencia y calidad de su trabajo, en el marco de una política de desarrollo sustentable.

El trabajo para el estudio, que incluye como dos grandes componentes la pesca artesanal y el turismo, se basó en los siguientes procedimientos:

- reuniones con autoridades solicitantes del estudio (directores y funcionarios de servicios públicos de la Región de Atacama);

- búsqueda de información documental;

- visitas a terreno a todo el litoral del BCA;

- referenciar geográficamente lugares de interés mediante GPS;

- aplicación de encuestas a pescadores;

- reuniones con representantes del sector turístico y de pescadores artesanales;

- talleres de consenso de propuestas con pescadores artesanales y con autoridades sectoriales.
Entre los productos que este estudio, en su conjunto, dejó para la autoridad demandante, están:

- Documento Informe del Estudio, que incluye:

a) Diagnóstico del Sistema Borde Costero (Pesca Artesanal y Turismo)

b) Línea base del sistema (resultados a alcanzar y componentes del sistema);

- $\mathrm{CD}$ interactivo que relaciona variables, lista datos y establece relaciones entre distintos elementos del sistema (este $\mathrm{CD}$ incluye una presentación cartográfica en Sistema de Información Geográfico - SIG);

- CD con fotografías de la infraestructura actual y los atractivos turísticos del BC;

- base de datos de atractivos turísticos; empresas turísticas y asociaciones y cámaras de turismo;

- listado de oportunidades actuales y potenciales para el sector;

- oportunidades seleccionadas;

- plan estratégico de intervención para el Sistema Borde Costero.

En la presente versión se presentan algunas informaciones tanto del Diagnóstico del Sistema Borde Costero, en la parte de turismo, como de la Línea Base del mismo sector, así como las oportunidades seleccionadas y algunas conclusiones de los análisis realizados como parte del estudio (Análisis FODA y análisis de encuestas, por ejemplo).

\section{Contexto de estudio: la Región de Atacama}

La Región de Atacama se ubica en el centro-norte de Chile, con una extensión de 75.573 kilómetros cuadrados, lo que equivale a 10\% de la superficie del país. Según el Censo Nacional de Población y Vivienda de 2002, realizado por el Instituto Nacional de Estadísticas - INE, la población regional alcanza a 254.336 habitantes, con una densidad poblacional de 3,4 habitantes por kilómetro cuadrado. La población regional representa al 1,7\% de la población nacional (15,1 millones de habitantes). La población urbana regional alcanza a 232.619 habitantes y la población rural es de 21.717 habitantes. La Región de Atacama se ubica entre los paralelos $25,17^{\circ}$ y $29^{\circ}$ latitud sur, teniendo como límite oriental la República Argentina y al occidente el Océano Pacífico, con un litoral de más de 500 kilómetros.

De acuerdo con el documento oficial Estrategia de Desarrollo Regional para la Región de Atacama (EDR, 2000), la minería constituye el principal sector 
económico de la región, seguido por la agricultura y la actividad pesquera y portuaria. El turismo no es una actividad económica fundamental.

\section{El turismo en Atacama}

La Región de Atacama presenta un incipiente desarrollo turístico basado en una variedad de atractivos naturales, culturales y actividades programadas, que debieran situarla, a mediano y largo plazo, en un destino importante para el turismo receptivo nacional e internacional. Desde el punto de vista del sistema turístico (Boullón, 1991), Atacama posee recursos tanto en sus 528 kilómetros de borde costero como en sus valles y en la precordillera y cordillera, así como también en las principales ciudades de la región, lo que representa una oferta potencial para diferentes tipos de turismo, incluyendo el de intereses especiales.

También se puede tener en cuenta la definición de patrimonio turístico para entender mejor el concepto de sistema turístico. Según Boullón (1991), el patrimonio turístico está formado por: a) atractivos turísticos; b) planta turística; c) infraestructura; y d) superestructura turística. Ambos conceptos (sistema y patrimonio turísticos) pueden interpretarse teniendo como base real al Borde Costero de la Región de Atacama.

Atendiendo a que ese proyecto trata sobre el borde costero, para el turismo no es posible segmentar absolutamente por sectores ya sean de carácter administrativo (comunas) o geográficos, debido a que los turistas no hacen ese tipo de diferencias y porque en general la oferta turística (al menos la planificada) tiende a integrar el territorio (costa, valles y cordillera) a través de diversos circuitos.

La Política Nacional de Uso del Borde Costero (Diario Oficial, 11.01.1995) define el Borde Costero del Litoral de Chile, como

aquella franja del territorio que comprende los terrenos de playa fiscales situados en el litoral, la playa, las bahías, golfos, estrechos y canales interiores, y el mar territorial de la República, que se encuentran sujetos al control, fiscalización y supervigilancia del Ministerio de Defensa Nacional, Subsecretaría de Marina.

El mismo documento define el ámbito de planificación de la ley y señala que ésta se aplicará en:

a) terrenos de playa fiscales ubicados dentro de una franja de 80 metros de ancho, medidos desde la más alta marea de la costa del litoral; b) la playa;

c) las bahías, golfos, estrechos y canales interiores, y;

d) el mar territorial de la República.

Sin embargo, en el presente proyecto, para el caso del turismo, entenderemos borde costero o franja costera una franja irregular de tierra que va desde la línea de costa (hasta donde llegan las aguas del mar) hasta los $300 \mathrm{msnm}^{*}$ en dirección Este. Esta definición divide el territorio de la Región de Coquimbo (y a nuestro juicio, extensivo a la de Atacama) en cuatro grandes unidades geográficofísicas postuladas por Paskoff (1970) y citadas por Véliz (1995): alta montaña (más de $600 \mathrm{msnm}$ ); montaña media (300 a $600 \mathrm{msnm}$ ); franja costera ( 0 a 300 $\mathrm{msnm}$ ) y valles transversales ( 0 a más de $600 \mathrm{msnm}$ ). Asumimos esta definición, más amplia en cuanto a superficie, puesto que los estrechos 80 metros desde la línea de costa a la más alta marea impedirían por definición la realización de gran parte de las actividades de un sistema turístico.

El ex intendente regional, Eduardo Morales (1996), señala como borde costero

el terreno que está comprendido desde la línea de las más bajas mareas hasta el primer cordón de cerros, más o menos desde 0 hasta los $300 \mathrm{msnm}$, lo que nos da una zona regulada que no es homogénea, en algunas partes es muy estrecha y en otras partes se abre y penetra hasta 30 kilómetros en el territorio $[\ldots]$

Si pensamos el turismo solamente para el borde costero, esta oferta se observa bastante reducida, pero si vemos la región como un todo se entenderá la amplitud de la oferta: a decenas de playas y balnearios marítimos, donde se puede practicar el tradicional turismo de sol y playa, Atacama suma ofertas relacionadas con el ecoturismo en parques nacionales como Pan de Azúcar, Llanos del Challe y Nevado de Tres Cruces; el turismo aventura en ascenso al volcán Ojos del Salado y los raids, rallys y sanboard; agroturismo y turismo rural en sus dos principales valles (Copiapó y Huasco); y otras particulares ofertas locales como el desierto florido, la gastronomía, la artesanía y el folclore.

Cualquier análisis que se haga del turismo en la región dará cuenta de cifras globales para el sector y en ningún caso para un subsector como el borde costero, a diferencia de lo que ocurre con otras actividades como la pesca o la acuicultura.

* Metros sobre el nivel del mar. [N.R.] 
La perspectiva de crecimiento del turismo regional se confirma con importantes proyectos que están en diferentes etapas de su planificación, y entre los cuales es necesario destacar la construcción de la ruta costera entre Huasco y Caldera; la construcción del nuevo aeropuerto internacional en Caldera; el proyecto Global Environment Fund (GeF) de Área Marina Protegida de múltiples usos; el Proyecto turístico-inmobiliario de Bahía Cisne y el plan de obras portuarias.

A la potencialidad presente de los recursos turísticos de Atacama y a su potencialidad futura, dadas las expectativas generadas con los proyectos mencionados, es necesario sumar ciertas limitaciones y debilidades de la región para enfrentar su desarrollo turístico. Algunas de estas limitaciones han sido reconocidas por sus autoridades sectoriales $y$, por lo mismo, se trabaja en superarlas, pero se requiere del concurso de fuerzas públicas y privadas para su superación. A seguir se enuncian algunas de esas limitantes:

- escasa proyección de la oferta local a nivel nacional e internacional;

- marcada estacionalidad de la actividad, concentrada en los meses de verano;

- falta de asociatividad entre los empresarios locales;

- el turismo no se ha posicionado como actividad importante para el desarrollo regional;

- falta de estructuración de un producto turístico regional;

- escasez de recursos para la promoción y difusión.

\section{Un desarrollo incipiente}

El carácter incipiente del desarrollo turístico regional se aprecia en la llegada de turistas extranjeros (larga distancia) a la región respecto del nivel nacional. Los turistas extranjeros que llegaron a Chile en 2001 a nivel nacional fueron 1.723.107 personas, mientras que los llegados a la Región de Atacama alcanzaron apenas a 3.585 personas. En Chile, la Región Atacama es la tercera menos visitada por extranjeros, después de la VII (31) y la VIII (621), según datos oficiales de 2001.

La llegada de turistas a la región aunque está en aumento ha tenido un crecimiento irregular, lo que puede observarse, entre otros datos, por informaciones del Instituto Nacional de Estadísticas - INE, dela Corporación Nacional Forestal - CONAF y del Servicio Nacional de Turismo - SERNATUR. La llegada de pasajeros; el número de pernoctaciones en establecimientos de alojamiento turístico; las visitas a parques nacionales y otras áreas protegidas; y el número de consultas a oficinas de información turística, son algunos de los indicadores que muestran este aumento.

Ese crecimiento no sostenido de la demanda puede verse claramente en las visitas al Parque Nacional Pan de Azúcar, las que aumentaron en 200\% entre 1994 (5.047) y 1997 (15.316) para caer en casi 50\% entre 1997 y 2001 (10.625).

La irregularidad en la demanda turística regional puede observarse también en otras cifras, tales como las pernoctaciones de pasajeros en establecimientos de alojamiento turístico (Tabla 1).

Tabla 1 Pernoctaciones de pasajeros en establecimientos de alojamiento turístico

\begin{tabular}{cccccc}
\hline Año & $\begin{array}{c}\text { Total país } \\
\left(\mathbf{n}^{\mathbf{0}}\right)\end{array}$ & $\begin{array}{c}\text { Variación país } \\
(\%)\end{array}$ & $\begin{array}{c}\text { Atacama } \\
\left(\mathbf{N}^{\mathbf{0}}\right)\end{array}$ & $\begin{array}{c}\text { Participación } \\
(\%)\end{array}$ & $\begin{array}{c}\text { Crecimiento } \\
\text { Región }\end{array}$ \\
\hline 1995 & 5.828 .508 & - & 227 & 0.00390 & - \\
1996 & 6.700 .510 & 14,96 & 237 & 0.00354 & $-9,12$ \\
1997 & 7.282 .471 & 8,69 & 242 & 0.00332 & $-6,27$ \\
1998 & 7.058 .121 & $-3,08$ & 206 & 0.00292 & $-11,94$ \\
1999 & 5.807 .811 & $-17,71$ & 176 & 0.00304 & 3,93 \\
2000 & 6.107 .441 & 5,16 & 177 & 0.00290 & $-4,42$ \\
2001 & 5.784 .149 & $-5,29$ & 169 & 0.00293 & 0,89 \\
\hline
\end{tabular}

Fuente: INE, SERNATUR. Anuario de turismo 2001

Uno de los datos más difíciles de cuantificar en turismo es la mano de obra empleada debido, entre otras razones, a la estacionalidad de la actividad, que se concentra en los meses de verano. Según datos de SERNATUR y de acuerdo a la medición de las actividades características del turismo de 1999 - que hace mediciones en temporada alta (ТА) y temporada baja (ТВ) -, para la Región de Atacama el empleo en el sector es de 4.942 personas en TA y de 3.334 en TB. Estos números muestran una participación regional de $2 \%$ de los ocupados en actividades características del turismo en el nivel nacional.

SERNATUR Atacama ha definido una serie de nueve lugares turísticos relevantes en la región. El carácter relevante tiene para SERNATUR dos sentidos. Uno: que en esos lugares hay una prioridad para futuros inversionistas. Esta prioridad fue dada en función de los recursos de esas zonas, su atractivo turístico y su potencial 
desarrollo en función de diversas características, como accesibilidad, infraestructura y servicios básicos. Y dos: que estos lugares son relevantes también para los turistas, es decir, la promoción turística de la Región de Atacama se hará destacando, en mercados nacionales e internacionales, estos nueve destinos.

Del total de nueve áreas prioritarias para el desarrollo del turismo en la Región de Atacama, según el criterio de SERNATUR, tres de ellas que son de primera prioridad están situadas en el borde costero, mientras que otras dos del mismo sector son de segunda prioridad. Se trata de zonas de playas y caletas (aldeas de pescadores) que ya cuentan con un cierto nivel de infraestructura turística y que se caracterizan por un fuerte atractivo en cuanto a paisajes y actividades relacionadas con el mar.

\section{Componentes del sistema}

En la franja costera y como parte del sistema turístico de Atacama tanto para la elaboración del Diagnóstico y Línea de Base del sistema así como para la elaboración consensuada de propuestas para el desarrollo turísticoteniendo como base las organizaciones de pescadores, se han identificado una serie de componentes principales:

a) caletas;

b) playas;

c) áreas silvestres protegidas y otras similares;

d) empresas y servicios turísticos (alojamiento, alimentación, agencias y cámaras);

e) atractivos en general (incluye circuitos turísticos y lugares para pesca deportiva);

f) asociaciones y cámaras de turismo.

La descripción detallada de estos seis componentes principales, realizada en el estudio original, se ha excluido de esta presentación por razones de espacio.

\section{Análisis de las encuestas aplicadas a pescadores en sus caletas}

El instrumento elaborado por SERCOTEC Y SERNATUR y aplicado por miembros del proyecto constaba en total de 77 preguntas, algunas de ellas desglosadas en varios ítemes. Hay preguntas cerradas (respuestas binarias) y preguntas abiertas.
Se aplicó un total de 15 encuestas en 13 de las 22 caletas. El instrumento aplicado fue respondido por la gran mayoría de las caletas que tienen población permanente y que cuentan con pescadores afiliados oficialmente. De acuerdo con los registros de SERNAPESCA, las caletas en que se aplicó la encuesta reúnen a 1.879 personas, de un total de 1.936, lo que significa que más de $95 \%$ de los pescadores registrados fue cubierto por la encuesta. En aquellas caletas con mayor número de pescadores (Caldera y Huasco) se aplicaron dos encuestas a cada una, respondidas por diferentes organizaciones.

Nueve caletas no fueron cubiertas por la encuesta porque no se encontró quien respondiera en el momento de aplicación del instrumento.

Para el análisis de esta encuesta se han cuantificado los resultados teniendo dos tipos de respuestas. Por un lado, para algunos datos que son más de percepción se consideró un total de 15 respuestas (todas las encuestas respondidas), mientras que en relación con datos más objetivos (referidos a infraestructura, por ejemplo) se contabilizó sólo 13 de las 15 respuestas. Los porcentajes están tomados sobre la base de esos totales.

Unalectura simple dela tabla de tabulación dela encuesta permite destacar algunos de sus principales resultados. Se presentan en forma sintética por razones de espacio:

1. Los turistas llegan a la caleta a lo largo del año y en una gran cantidad;

2. Existe una percepción positiva del turismo;

3. Hay una alta demanda de servicios de parte de los turistas a los pescadores;

4. Hay una visión positiva del desarrollo local en el BCA;

5. Las caletas cuentan con algunos servicios o equipamientos básicos tales como:
a) teléfono: $54 \%$
b) señal de Tv: $62 \%$
c) señales de radioemisoras: $77 \%$;

6. Hay una visión positiva del papel de las instituciones públicas en el BCA;

7. Sin embargo, hay una serie de elementos negativos o limitantes para el desarrollo del turismo en el $\mathrm{BCA}$, según las encuestas, teniendo como eje a las caletas de pescadores:

a) un $46 \%$ de las caletas encuestadas señala que hay familias que viven en la misma;

b) la gran mayoría de los visitantes a las caletas son familiares (93\%);

c) existe una baja oferta de servicios que los turistas solicitan, como:

- paseos en bote: $38 \%$

- paseos a caballo: $23 \%$ 
- otros servicios (venta de combustibles, mecánico, baños para turistas) son poco ofertados

d) Existe una muy baja relación entre pescadores y empresas turísticas ( $15 \%$ reconoce tener contactos)

e) $93 \%$ señala que los turistas alojan en carpas. Dato no exacto (pues se aceptan respuestas no excluyentes), pero significativo

f) $54 \%$ señala que la basura es un problema

g) $26 \%$ tiene salvavidas en verano

h) $38 \%$ cuenta con red de agua potable

i) $31 \%$ con red de energía eléctrica;

8. Los pescadores reconocen un patrimonio cultural que puede ser un atractivo turístico a desarrollar en diversos aspectos;

9. Otro dato de interés es que $47 \%$ dice que los pescadores se dicen muy organizados; $33 \%$ poco organizados, y $13 \%$ dice que están divididos;

10. A juzgar por los pescadores, el ambiente en que viven y/o trabajan es altamente atractivo para el turismo ya que la mayoría señala que en ellos hay presencia o existencia de recursos turísticos tales como observación y anidación de aves; loberías o chungungos; observación de delfines o toninas; sitios arqueológicos; y cercanías de naufragios de barcos, entre muchos otros.

\section{Evaluación y clasificación de la potencialidad turística de las playas del BCA}

Tanto las playas, desde su atractivo turístico, como las caletas desde el punto de vista de su importancia económica y social, fueron sometidas a una evaluación de sus potencialidades en función de una serie de variables, con el objetivo de hacer una tabla de prioridades.

En el caso de las playas se tuvo en cuenta las siguientes variables:

1. accesibilidad

2. tendido eléctrico y agua potable

3. camping con baños

4. servicios turísticos (hospedaje, restaurante y otros)

5. contaminación de las playas (se refiere a basuras, olores y otros efectos visibles)
6. belleza paisajística

7. área priorizada por SERNATUR (según el mapa de lugares turísticos relevantes y otras indicaciones)

8. habilitación oficial (playa apta o no apta, según Gobernación Marítima)

Como resultado de esta evaluación, las playas con mayor potencial para el desarrollo turístico en el BCA son: Pan de Azúcar, Chañaral, Caldera, Carrizal Bajo, Huasco y Chañaral de Aceituno.

\section{Esbozo de propuestas de intervención}

A través del análisis de la medición del potencial de cada zona de interés en función de las diversas variables, tanto el aspecto de pesca como de turismo, se obtuvo como resultado la clasificación de las 22 caletas en diversos grupos de prioridad. El primer sector, integrado por Caletas Caldera, Chañaral, Huasco y Carrizal Bajo, es el que presenta mejores condiciones conjuntamente en turismo y en pesca artesanal, y por tanto son las caletas de primera prioridad para la ejecución de planes de intervención.

A estegrupo prioritario, aparentemente, resulta conveniente incluir dos caletas del segundo grupo, debido a su organización y proyección en el aspecto turístico. Dichas caletas son Pan de Azúcar (cuenta con un parque nacional lo que la hace atractiva turísticamente); y el sector de Chañaral de Aceituno debido a su gran potencial pesquero y turístico, pero principalmente por ser una caleta muy bien organizada. En cualquier caso, en las restantes caletas/sectores a continuación se propusieron proyectos transversales de fortalecimiento organizacional y de capacitación.

A continuación se señalan los planes preliminares de intervención (para sectores de pesca y turismo) en las caletas priorizadas y con un horizonte de tiempo de tres años:

Renovación, modernización e implementación de equipos e infraestructura

- Construcción e implementación de sistema de frío.

- Reemplazo de embarcaciones antiguas.

- Implementación de tecnología de apoyo a la pesca (GPS y ecosonda). 


\section{Complementos al turismo}

- Instalación de museo y biblioteca.

- Implementación de botes de paseo.

- Habilitación de servicios de alimentación.

- Habilitación de servicios de alojamiento.

\section{Diversificación productiva}

- Implementación y capacitación en nuevos artes de pesca.

- Implementación de una pequeña planta de proceso.

- Implementación de pequeños centros de cultivos acuícolas.

\section{Capacitación}

- Capacitación como guías turísticos.

- Manipulación de alimentos.

- Capacitación en gestión de microempresas.

\section{Transversales}

- Fortalecimiento organizacional.

- Solución a los conflictos de propiedad.

\section{Breve descripción de proyectos específicos para el sector Turismo}

Específicamente para el sector de turismo se describen algunos de esos proyectos:

\section{Instalación de museo - Biblioteca}

La finalidad de construir un museo - biblioteca es que exista un lugar donde se pueda apreciar la cultura del pescador y el avance en las técnicas que estos han empleado en el tiempo, además de contar con apoyo bibliográfico. En un principio este museo biblioteca se construiría en Carrizal Bajo, y si el modelo resulta exitoso se aplicaría a otras caletas de la región.
A través de fotografías, mapasy un video del entorno de la caleta - apuntando a su flora y fauna terrestre -, y con la exposición de las artes de pesca usadas por pescadores de la zona, de la flora y de la fauna marítima (materiales reales o réplicas y fotografías, mapas y videos), el visitante podrá aprender, con mayor o menor profundidad, qué es un área de manejo, qué son los cultivos marinos, qué tipos de pesca se realizan en ese lugar, qué pesca deportiva se puede practicar.

Su objetivo principal es difundir (y preservar) la cultura del pescador. Por ello, una parte de la muestra es la artesanía local, aquella producida por los pescadores y sus familiares con productos típicos de la zona. El museo servirá para desarrollar una parte de la actividad educativa, tanto para los turistas como para estudiantes de la región. La componente educativa en lo ambiental puede desarrollarse partiendo de este contacto. El museo puede tener anexado un local de venta de artesanías y otros recuerdos (fotos postales de la caleta, etc.) Una de sus posibles ampliaciones es la construcción de acuarios, por ejemplo.

\section{Diseño de plan de negocios para turismo con fines especiales}

Existe una serie de alternativas relacionadas con el turismo que pueden ser adoptadas por los pescadores y sus familias. Para esto se requiere del diseño de un plan de negocios para presentar una oferta de turismo con fines especiales. La idea es también transformar a los pescadores en sujetos de crédito. Formar sociedades de pequeños grupos, por ejemplo, de dueños de botes o lanchas que se dediquen a esta actividad. Este proyecto se puede implementar en Chañaral, Caldera, Huasco, Pan de Azúcar, Carrizal bajo y Chañaral de Aceituno:

a) paseos en botes y lanchas;

b) habilitación de servicios de alimentación;

c) habilitación de servicios de alojamiento;

i) establecer una oferta de alojamiento;

ii) mejorar la oferta de alojamiento.

\section{Capacitación a pescadores como guías de turismo}

Los pescadores o sus familias pueden capacitarse con nociones básicas de turismo, geografía e historia de la región. Un curso de 20 horas como mínimo, al cual se pueden añadir otras 20 horas para un curso básico de inglés. El curso incluye una salida a terreno, con un circuito predeterminado y la presentación de los lugares por los alumnos (como evaluación formativa). 


\section{Capacitación en gestión de microempresas}

Las organizaciones base de la mayor parte de las caletas se caracterizan por una estrategia organizativa que responde a objetivos sociales, lo que las condiciona como productores informales sin capacidad de autogestión y de rentabilizar su producción, puesto que carecen de gestión comercial propia. En este contexto, para intervenir su estructura productiva es necesario potenciar su estrategia comercial, modificando su estructura organizacional a una figura legal que responda a objetivos comerciales; capacitar en elámbito del negocio (gestión de negocios), y potenciar sus factores de producción (embarcaciones y sistemas de pesca).

La capacitación en gestión de microempresas permitirá a los pescadores beneficiados contar con herramientas para desenvolverse en el ámbito empresarial a pequeña escala, es decir, como microempresarios. Esta capacitación logrará que los pescadores desempeñen y conozcan funciones administrativas básicas como costos y leyes tributarias a fin de tener conocimientos básicos que les permitan manejar eficientemente sus asociaciones; además esta capacitación permitirá que sean los propios pescadores quienes administren los servicios turísticos de sus caletas.

\section{Solucionar conflictos de propiedad}

Resulta importante destacar que los conflictos de propiedad son un problema que atañe directamente a pescadores y sus familias, ya que varias de las ocupaciones ilegales de terrenos en la Región de Atacama se encuentran en las cercanías de las caletas. Bienes Nacionales es el organismo encargado de regularizar este tipo de situación, pero esta solución puede tardar mucho tiempo,especialmente en ocupaciones de terrenos particulares dado que sus dueños no están de acuerdo en vender. La solución es un plan de regularización de títulos de dominio lo más rápido posible; esta situación tendría un gran impacto en los pescadores y sus familias. Los principales sectores afectados son las caletas Flamenco, Puerto Viejo, Barranquilla, Pajonales, Carrizal Bajo y Chañaral de Aceituno.

\section{Programa de fortalecimiento organizacional}

Uno de los graves problemas que surge en las caletas de la Región de Atacama se refiere al poco nivel organizacional que presentan los sindicatos y otras entidades. Las asociaciones gremiales de pescadores no han tenido capacitación en fortalecimiento organizacional que les permita mejorar sus liderazgos y obtener conocimientos de fuentes de financiamiento o formalidades jurídicas. Por esta razón este proyecto se orienta a crear un programa de fortalecimiento organizacional compuesto por:

- formación de líderes comunitarios;

- agenda de desarrollo de organizaciones de base;

- seguimiento de reuniones.

\section{Programa de fortalecimiento organizacional para asociaciones de guías de turismo}

De cinco asociaciones de guías existentes, tres de ellas fueron creadas en 2002: Agrupación de Guías de Ecoturismo, Turismo Aventura y Patrimonio Cultural de la Región de Atacama; Agrupación Provincial (Chañaral) de Guías de Turismo; y Agrupación Guías Turismo de la Provincia del Huasco. Las otras dos son un poco anteriores: Agrupación de guías de turismo de la Provincia de Chañaral (1998) y Grupo de Patrimonio Cultural de Comuna de Freirina (2001).

Por esta razón este proyecto se orienta a crear un programa de fortalecimiento organizacional, en una primera etapa, y una vez logrado ese objetivo, estas organizaciones podrán capacitarse para la creación de circuitos turísticos en turismo de intereses especiales y como guías especializados en esos circuitos.

La primera etapa, de fortalecimiento organizacional, debe estar centrada en:

- formación de líderes (importancia de la organización gremial en la sociedad actual; papel del líder; tipos de liderazgo y tipos de organizaciones; la sustentabilidad de la organización gremial);

- agenda de desarrollo de organizaciones (importancia de la personalidad jurídica; autonomía para la formulación y ejecución de proyectos; conocimiento y acceso a fuentes de financiamiento de proyectos; beneficios para los asociados, deberes y derechos de los miembros).

La segunda etapa estará dedicada a:

- crear, con apoyo de especialistas, circuitos turísticos para el turismo de intereses especiales, integrando las diversas áreas geográficas de la Región de Atacama (Costa, Valles, Montaña) y sus diversos atractivos (naturales y culturales), con especial énfasis en las actividades de los pescadores en el Borde Costero; 
- capacitar a los guías turísticos especializados en estos circuitos, con conocimientos específicos de historia, geografía y turismo sustentable.

\section{Programa de fortalecimiento organizacional para cámaras de turismo}

Las cámaras de turismo de la región (Provincial de Turismo de Copiapó; Asociación de Cámaras de Comercio y Turismo de Chañaral y Cámara de Turismo de Caldera), son también organizaciones que necesitan de mayor desarrollo y fortalecimiento como entidades gremiales.

En el caso de estas organizaciones, es posible llevar adelante un proyecto de fomento (PROFO) con financiamiento estatal (corporación de fomento) para la elaboración y promoción de un producto turístico integrado conénfasisen los atractivos del Borde Costero.

En el Diagnóstico del Sector Turismo para la Región de Atacama, hecho por las autoridades locales, se reconoce que una de las debilidades es la noexistencia de un Producto Turístico Integrado.

Antes de promover un destino turístico, éste debe ser un destino con un producto turístico idealmente integrado, que constituya una oferta única pero que contenga una serie de atractivos: lugares, servicios, actividades, etc.

\section{Reserva marina en el sistema insular de Isla Chañaral}

La creación de esta reserva marina en la Isla Chañaral, con participación activa de los pescadores, tendría los siguientes objetivos:

- protección de la biodiversidad asociada a la isla Chañaral;

- proporcionar los medios para el desarrollo del ecoturismo y establecer las oportunidades para el desarrollo de un negocio turístico con base a la observación de poblaciones de mamíferos y aves marinas con categoría de observación;

- proveer oportunidades para el desarrollo de educación ambiental dirigidos a la comunidad para contribuir a la valoración integral de medio ambiente marino;

- establecer las oportunidades para la investigación y monitoreo de especies símbolos y sistemas biológicos para aumentar el conocimiento sobre su estructura, organización y dinámica, dado que forman parte de un patrimonio regional de desarrollo pesquero (mamíferos y aves marinas con categoría de conservación, y peces con interés comercial).

\section{Conclusiones}

A pesar del interés creciente en el sector turismo en la región, falta el desarrollo en la práctica de una política regional de turismo, cuyas bases ya han sido trazadas por las autoridades regionales. La perspectiva de crecimiento del turismo regional se confirma con importantes proyectos que están en diferentes etapas de su ejecución ya mencionados.

El BCA es un territorio cuyo principal atractivo turístico es de carácter natural (playas y áreas naturales) con un componente humano no siempre presente (los pescadores en sus caletas) y un número reducido de servicios y empresas prestadoras de servicios. En este ambiente natural, cuyo clima es conveniente para el turismo (escasez de lluvias, temperaturas moderadas), se presenta una variedad de atractivos que permiten la realización de actividades que pueden satisfacer una diversidad de motivaciones turísticas.

\section{Referencias bibliográficas}

BOULLÓN, R. 1991. Planificación del espacio turístico. Trillas, México.

CARABANTES, J. 2000. Plan de desarrollo y manejo del ecoturismo en el Parque Nacional de Llanos de Challe y alrededores. Universidad de La Serena.

DIARIO OFICIAL DE LA REPÚBLICA DE CHILE. 1995. Política nacional de uso del borde costero. p. 3 y 4,11 de enero.

ERD. Estrategia Regional de Desarrollo Atacama. 2000. Visión global de futuro. Sector Turismo. Documento de trabajo de sernatur Región de Atacama.

LAZO, Alex. 1997. Plan de desarrollo turístico comunal de Huasco. Universidad de La Serena.

MANUAL de Atacama. Documento oficial Región de Atacama.

MORALES, E. 1996. La Región de Atacama y el medio ambiente costero. Primer Seminario Nacional Política Nacional de Uso del Borde Costero. Santiago.

PLAN de marketing (Borrador). 2002. Documento de trabajo de SERNATUR Región de Atacama. SERNATUR. 1996. Plan Maestro de Desarrollo Turístico para la Región de Atacama (Versión digital en SITERRA).

SERNATUR. Dirección nacional. 2001. Antecedentes básicos sobre pesca deportiva en Chile. Santiago SERNATUR-INE. 2000-2001. Anuarios de Turismo. Santiago de Chile.

SERnATUR REgión a atACAMA. (Sin fecha). Bases para una Política Regional de Turismo.

VÉLIZ, G. 1995. Conurbación La Serena-Coquimbo. Universidad de La Serena.

www.conaf.cl

www.directemar.cl

www.sernapesca.cl

www.sernatur.cl 
46 Turismo em Análise, v. 16, n. 1, p. 29-46, maio 2005

Documentos de trabajo de SERNATUR de Atacama:

- Estadísticas de visitantes

- Directorio de restaurantes y otros

- Manual de informaciones turísticas

- Listado de alojamientos turísticos

Recibido en 12/08/2004.

Aprobado en 28/09/2004. 